of modern concepts. Natural products are given representative treatment in separate chapters dealing with carbohydrates, terpenes, steroids, vitamins and alkaloids, while topics such as carotenoids, tropolones, penicillins, anthocyanins and pyrrole pigments are introduced as sub-sections in the general text. Throughout, the aim is to equip the reader for specialist reading and to guide him towards the fringes of current research.

The book provides an excellent refresher course : it will also be widely consulted for its stereochemistry. Although the authors disclaim the intention of writing an examination text-book, the honours student can scarcely fail to find it a profitable study. Yet it requires critical reading, for on occasion the authors reach or imply conclusions which are not strictly warranted by the facts presented. For example, the conclusion that certain optically active alcohols which have the same rotational sign have also the same configuration does not follow from the context on p. 637 ; nor is it there explained what meaning is to be attached to "same configuration" as applied to benzylmethylcarbinol and ethyl $\beta$-hydroxy- $\beta$-phenyl-propionate. None the less, the book is written well and in a manner which captures and sustains interest. Despite its size - it is somewhat unwieldy-and the complexity of the formulæ, it is remarkably free from errors of printing. But one cannot commend the practice--here inconsistently applied - of denoting a ring-member by a symbol placed beside the ring-outline as if it were a substituent. In the alicyclic series this is an unsightly and unnecessary elaboration; in the heterocyclic series, where a valency count may not remove the ambiguity, it is a real and avoidable source of misunderstanding.

J. D. Loucon

\section{STRUCTURE-ACTIVITY CORRELATION IN THE MAKING} First Symposium on Chemical-Biological Correlation, May $26-27,1950$

Sponsored by the Chemical-Biological Coordination Center of the National Research Council. Pp. $v+415$. (Washington, D.C. : National Academy of Sciences/ National Research Council, 1951.) 4 dollars.

CINCE Crum-Brown and Fraser in 1869 ventured $\$$ to present the relationship between the chemical constitution of a drug $(C)$ and its pharmacological activity $(\varphi)$ in the form of a pseudo-mathematical equation $\varphi=f(C)$, numerous attempts have been made to verify this relatively simple expression. That these efforts to find a rational basis for drug action have not resulted in overwhelming success is due to various reasons : for example, the meaning of $C$, the chemical constitution, has often been accepted with superficiality as the two-dimensional formulæ used in chemists' notebooks and not as an integrated collection of all the finer points of physical and chemical properties of the compounds under consideration; differences in the mechanism of action between drugs achieving the same end-effects have been frequently ignored, and the existence of receptor molecules, possessing, like the drugs, specificity of structure and reactivity, has rarely been taken into account; differences in the degree of accuracy between the measurements of biological effects and certain physical-chemical characteristics have been overlooked in many cases.
It is therefore not surprising that, during the past thirty years or so, most of the major advances in the field of biologically active substances have been made by trial and error and by fortuitous discoveries. Yet, here and there, groups of therapeutics were found which were more amenable to a rational treatment, and the emergence of the theory of antimetabolite action has given renewed impetus to the slowly moving searches. But there still remains the necessity for more and more data of biochemical, physicochemical and biological observations, the last-named as quantitative in nature as possible.

To provide such data the United States National Research Council founded in 1946 the ChemicalBiological Coordination Center. With lively optimism, so characteristic of many American undertakings, this body has developed procedures for the collection of data sheets, the preparation of card files and the use of chemical and biological codes. From time to time, accumulated results are published in the form of booklets. True, the chemical data are sometimes scanty and physical-clectronic properties more than often missing. The biological results suffer from the fact that they belong to the plus-minus variety; but matters have progressed sufficiently to warrant, in May 1950, the arrangement of the first symposium on simply, perhaps over-simply, expressed "ChemicalBiological Correlation". The lectures and discussions were published during 1951, unpretentiously in varitype print, giving to everyone interested in the subject the chance to see for themselves how matters stand. There are a number of most stimulating contributions, such as that by Schubert on the effect of drugs on physiologically active thiol systems, and by Friedman on the influence of isosteric replacements upon biological activity. Others, experts in various special fields, like Doak and Eagle on arsenosobenzenes, Norman and Weintraub on plant growth-regulators, Lands on sympathomimeties, and Horsfall et al. on fungicides, have chosen a defined group of closely related substances to prove their case. Two lectures on antihistamines and antithyroids were used to demonstrate the procedures and the systems introduced by the Center and separately explained by its director, Dr. Kirner, and Drs. Beard and Geer. Two panels were discussing antimalarials and antimetabolites, carcinogenesis and cancer therapy. With regard to the first, one could quote F. L. Rose's statement on the occasion of his Tilden Lecture, "A Chemotherapeutic Search in Retrospect": ". . . the use of the "working hypothesis' concept based on observation and the intelligent development of leads . . . must constitute the foundation for speculative research in this field for a long time to come". As to the wide and complex subject of antimetabolites, carcinogens and carcinolytic substances, only the antimetabolites seem to obey certain rules. The rationalization of conditions of cytotoxic compounds is more than ever waiting for the discovery of their true mechanism of action. But this problem, although a very thorny one, and those problems connected with drugs where the mode of action has been more fully elucidated, may yield sooner than expected at present if the attempts at producing and then collecting all pertinent data are vigorously continued; for, as Hales said at the beginning of the eighteenth century, "hardly do we guess aright at the things that are upon earth, and with labour do we find the things that are before us". F. Berger 